\title{
Nuevas tecnologías en el procesamiento de recubrimientos de cerámicas bioactivas
}

\author{
E. L. SOLLA, J. P. BORRAJO, P. GONZÁLEZ, J. SERRA, S. LISTE, S. CHIUSSI, B. LEÓN, M. PÉREZ-AMOR \\ Universidad de Vigo, Departamento de Física Aplicada, Lagoas-Marcosende, 36200 Vigo.
}

\begin{abstract}
En la actualidad existen nuevas tecnologías de procesamiento de recubrimientos basados en la técnica denominada depósito por láser pulsado (PLD), la cual consiste en la ablación de un blanco mediante un haz láser y como consecuencia de la interacción radiación - materia se origina el transporte del material hasta el sustrato a recubrir. Esta técnica es muy versátil ya que permite la incorporación de atmósferas reactivas en el proceso y de descargas eléctricas en la pluma de ablación las cuales pueden inducir modificaciones en las propiedades de los recubrimientos.

En este trabajo se aplican estas nuevas tecnologías a la obtención de capas cerámicas bioactivas de hidroxiapatita y de vidrio bioactivo. Se ha estudiado la influencia de parámetros de proceso como temperatura, diferencia de potencial de la descarga de radiofrecuencia aplicada y atmósfera reactiva, desde el punto de vista de la composición y estructura de los recubrimientos. En el caso de la hidroxiapatita, la presencia de una atmósfera de vapor de agua y de una descarga de radiofrecuencia favorece el ritmo de depósito y la cristalinidad de los recubrimientos. Sin embargo, los recubrimientos óptimos de vidrio bioactivo se obtienen en condiciones de vacío y ausencia de descargas eléctricas.
\end{abstract}

Palabras clave: Ablación Láser, Descarga Radiofrecuencia, Cerámica Bioactiva, Atmósfera Reactiva.

\section{New technologies in the processing of hidroxyapatite and bioactive glass coatings}

Recently new coating technologies have been developed based on the so-called Pulsed Laser Deposition (PLD) method, which consists in the laser ablation of a target material, and as a consequence of the matter-radiation interaction, the transport of ablated species from the target to the substrate is originated. PLD is quite versatile technique, and allows the incorporation of a reactive atmosphere and electric discharges in the process so that the properties of the coatings can be modified.

In this work, these new technologies were applied in the processing of bioactive ceramic thin films of hydroxyapatite and bioactive glass. The influence of processing parameters, such as temperature, voltage applied of the radiofrequency discharge and reactive atmosphere, on the coating properties have been studied. It was found that in the case of hydroxyapatite coatings, the water vapour atmosphere coupled with the radiofrequency discharge enhances the deposition rate and cristallinity of the coatings. Nevertheless, the optimum properties for bioactive glass coatings were obtained in vacuum and absence of electrical discharge conditions.

Keywords: Laser Ablation, Radiofrequency Discharge, Bioactive Ceramics, Reactive Atmosphere.

\section{INTRODUCCIÓN}

Se denominan biomateriales aquellos considerados como sustancias no farmacológicas apropiadas para su inclusión en el medio biológico y que potencian o sustituyen las funciones de los órganos y tejidos corporales. Entre estos biomateriales se encuentran las cerámicas bioactivas y biovidrios, utilizados en los últimos años para mejorar la biocompatibilidad en implantes dentales y ortopédicos $(1,2)$; las cuales han jugado un papel muy importante por su interesante propiedad de reaccionar químicamente con el fluido biológico formando un enlace con el hueso vivo y el tejido conectivo blando, facilitando la regeneración ósea. En este grupo de materiales se encuentran la hidroxiapatita (HA), compuesto de tipo fosfato cálcico $\left(\mathrm{Ca}_{10}\left(\mathrm{PO}_{4}\right)_{6}(\mathrm{OH})_{2}\right)$ con una estructura y composición muy similar a la parte mineral del hueso (3), y los vidrios bioactivos. Las propiedades bioactivas de estos últimos fueron descubiertas en el año 1971 cuando Hench y col. (4) encontraron que vidrios dentro del sistema $\mathrm{SiO}_{2}-\mathrm{Na}_{2} \mathrm{O}-\mathrm{CaO}-$ $\mathrm{P}_{2} \mathrm{O}_{5}$ presentaban la capacidad de formar en su superficie una capa biológicamente activa al ponerse en contacto con fluidos corporales (5). Es interesante destacar que esta característica de los vidrios bioactivos viene generada por la discontinuidad en la matriz de $\mathrm{SiO}_{2}$, como consecuencia de la incorporación de los modificadores de red, dando lugar a la aparición de átomos de oxígeno no puente ( $\left.\mathrm{Si}-\mathrm{O}^{-}\right)$.

Los recubrimientos comerciales de este tipo de materiales se producen fundamentalmente mediante la técnica de pulverización por plasma (6), a pesar de que dichos recubrimientos presentan desventajas como su escasa adhesión al sustrato e inhomogeneidad en la composición (7). En los últimos años el depósito mediante ablación láser (Pulsed Laser Deposition, PLD) se ha revelado como una técnica muy prometedora en la producción de recubrimientos de hidroxiapatita (8), y vidrio bioactivo (9) ya que solventa las desventajas de la pulverización por plasma (10).

En el presente trabajo se aplican, en el procesamiento de hidroxiapatita y vidrio bioactivo mediante ablación láser, el uso de atmósferas reactivas y la aplicación de descargas eléctricas de radio frecuencia durante el proceso, así como 
también se analiza la influencia de estas modificaciones en las propiedades de los recubrimientos obtenidos.

\section{EXPERIMENTAL}

Para la obtención de los recubrimientos se utilizaron blancos de hidroxiapatita (HA) y vidrio bioactivo (VB) $\left(\mathrm{SiO}_{2}\right.$ $45 \%, \mathrm{Na}_{2} \mathrm{O} 24,5 \%, \mathrm{CaO} 24,5 \%, \mathrm{P}_{2} \mathrm{O}_{5} 6 \%$ ) obtenidos por la técnica clásica de fusión, los cuales fueron comprados a la Europa Abming Technology. En cuanto a los blancos de HA fueron obtenidos a partir de polvo de HA comercial (P120 Plasma Biotal) mediante prensado a $80 \mathrm{mPa}$, mientras que los depósitos fueron realizados sobre sustratos de Si. El proceso de ablación se realizó mediante un láser de excímero ArF (193 $\mathrm{nm}$ ) focalizado produciendo una densidad de energía de 4,2 $\mathrm{J} / \mathrm{cm}^{2}$ sobre la superficie de los blancos. Con el objeto de conseguir una erosión uniforme del blanco, éste permanece rotando a $3 \mathrm{rpm}$ durante todo el proceso de depósito. La producción del plasma de radiofrecuencia se llevó a cabo mediante un electrodo de cobre situado entre el blanco y el sustrato, pudiendo variar el voltaje de la descarga en un intervalo de 0 a $300 \mathrm{~V}$, manteniendo la frecuencia constante a $275 \mathrm{kHz}$.

Para estudiar la influencia de la atmósfera reactiva durante la ablación de HA, se realizaron experimentos en vacío y en presencia de atmósferas de vapor de agua (11) y Ar (ambas a $0,45 \mathrm{mbar}$ ). La temperatura del sustrato se fijó en $460^{\circ} \mathrm{C}$ por ser la temperatura a la cual se obtienen recubrimientos cristalinos (12-14). En cuanto a los recubrimientos de vidrio bioactivo, los depósitos fueron realizados tanto en vacío como en presencia de $\mathrm{NH}_{3}(0,05$ mbar) y Ar (0,45 mbar) (15) y la temperatura del sustrato fijada en $200{ }^{\circ} \mathrm{C}$, debido a que a esta temperatura, las propiedades de los recubrimientos son óptimas para aplicaciones biomédicas (16). Todos los depósitos tuvieron una duración de 15 minutos.

En cuanto a la caracterización de los recubrimientos, se realizó un estudio de los grupos activos en el infrarrojo mediante espectroscopía infrarroja con transformada de Fourier (FTIR), con un espectrómetro Bruker IFS28 en modo de transmisión y a una resolución de $4 \mathrm{~cm}^{-1}$. En el caso de la HA las fases cristalinas se estudiaron mediante difracción de rayos $\mathrm{X}(\mathrm{XRD})$ con un difractómetro Siemens D5000, con la línea $\mathrm{Cu} \mathrm{K} \alpha$ a 40 kV y 30 mA, utilizando una configuración de medida $\theta-2 \theta$. La relación $\mathrm{Ca} / \mathrm{P}$ en porcentaje atómico se llevó a cabo mediante espectroscopía por dispersión de energías de rayos $X$ (EDS) con un espectrómetro EDAX instalado en un microscopio electrónico de barrido Philips XL30. Dicho cálculo de la relación $\mathrm{Ca} / \mathrm{P}$ se llevó a cabo calibrando con tres materiales de referencia de concentración $\mathrm{Ca} / \mathrm{P}$ conocida.

\section{ANÁLISIS DE RESULTADOS}

\subsection{Recubrimientos de hidroxiapatita}

En la Fig. 1 se muestran los espectros FTIR (Fig. 1) correspondientes a los recubrimientos de HA obtenidos mediante PLD sin plasma RF en vacío, atmósfera de Ar y vapor de agua. Dichos espectros presentan las principales bandas de absorción asignadas a los grupos funcionales de la HA, a saber: i) vibraciones correspondientes a la tensión (s) simétrica y antisimétrica $\left(1000-1200\right.$ y $\left.960 \mathrm{~cm}^{-1}\right)$ y a la flexión (b) antisimétrica $\left(560 \mathrm{~cm}^{-1}\right)$ de los grupos $\mathrm{PO}_{4}^{3-}$, y ii)

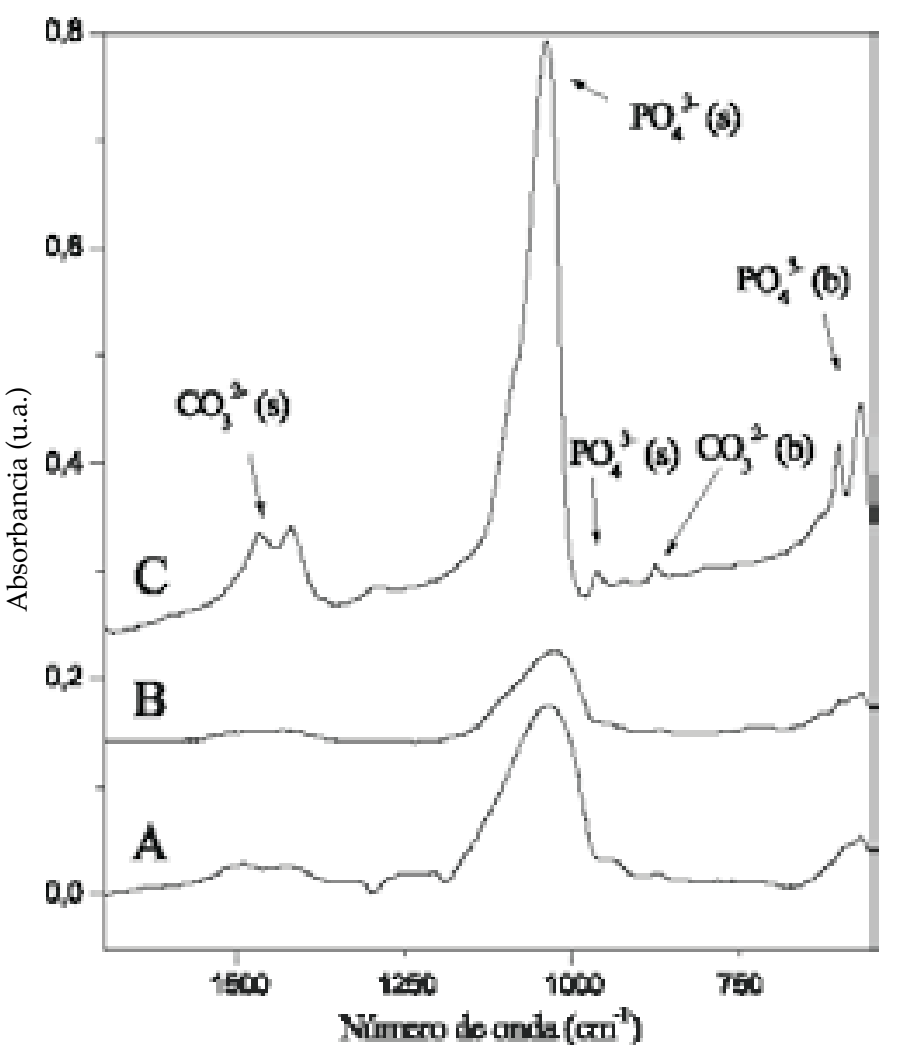

Fig. 1- Espectros FTIR de recubrimientos de HA depositados en: A) vacío, B) atmósfera de Ar y C) atmósfera de vapor de $\mathrm{H}_{2} \mathrm{O}$.

vibraciones correspondientes a la tensión antisimétrica (1400 - $\left.1500 \mathrm{~cm}^{-1}\right)$ y flexión $\left(875 \mathrm{~cm}^{-1}\right)$ de los grupos $\mathrm{CO}_{3}{ }^{2-}$. Como se puede observar, los cambios más significativos tienen lugar cuando los recubrimientos se obtienen en atmósfera de vapor de agua, pudiendo resumirlos de la siguiente forma: i) incremento en el ritmo de depósito indicado por el aumento de intensidad de las bandas de IR, ii) resolución de la banda ancha a $565 \mathrm{~cm}^{-1}$, correspondiente a los grupos $\mathrm{PO}_{4}^{3-}$ , en dos picos bien definidos característicos de compuestos apatíticos (17), iii) desplazamiento del máximo de absorción de la banda correspondiente a la tensión antisimétrica de los grupos $\mathrm{PO}_{4}^{3-}$ hacia mayores números de onda, poniendo de manifiesto cambios estructurales en la red cristalina, iv) aparición de un hombro en el doblete a $565 \mathrm{~cm}^{-1}$ asignado a grupos de $\mathrm{OH}^{-}$debido a la incorporación de agua en el recubrimiento, indicando un aumento de la cristalinidad en los recubrimientos (18), y v) resolución de la banda de tensión asimétrica de los grupos $\mathrm{CO}_{3}{ }^{2-}$ en dos bandas a 1496 y $1429 \mathrm{~cm}$ ${ }^{1}$, así como una mejor definición de la flexión fuera del plano de estos mismos grupos. Debido a estas razones, la atmósfera de vapor de $\mathrm{H}_{2} \mathrm{O}$ fue elegida como la más apropiada para la obtención de recubrimientos de HA por la clara mejoría, respecto a los recubrimientos obtenidos en Ar y vacío, en ritmo de depósito y cristalinidad.

Con el fin de estudiar la influencia de la descarga de RF en el proceso de ablación de la hidroxiapatita, se llevaron a cabo depósitos en atmósfera de vapor de agua variando el voltaje aplicado en el electrodo. La Fig. 2 muestra los espectros de IR de los recubrimientos obtenidos a diferente voltaje de RF, los cuales presentan las ya anteriormente mencionadas bandas de absorción relativas a la hidroxiapatita carbonatada. Considerando el espectro obtenido a $0 \mathrm{~V}$ (Fig. 2-a) como material de referencia, es evidente que la estructura 


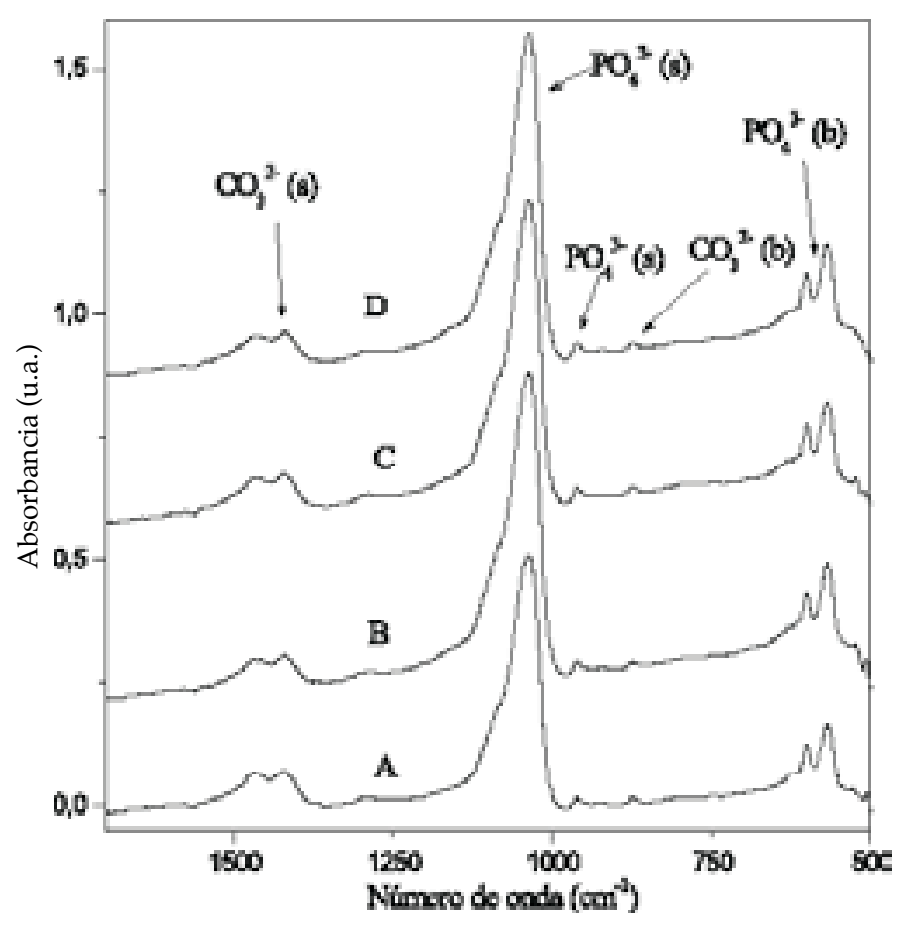

Fig. 2- Espectros FTIR de recubrimientos de HA depositados en atmósfera de vapor de $\mathrm{H}_{2} \mathrm{O}$ con descarga de $\mathrm{RF}$ a distintos voltajes: A) $0 \mathrm{~V}, \mathrm{~B}$ ) $247 \mathrm{~V}, \mathrm{C}) 265 \mathrm{~V}, \mathrm{D}) 300 \mathrm{~V}$.

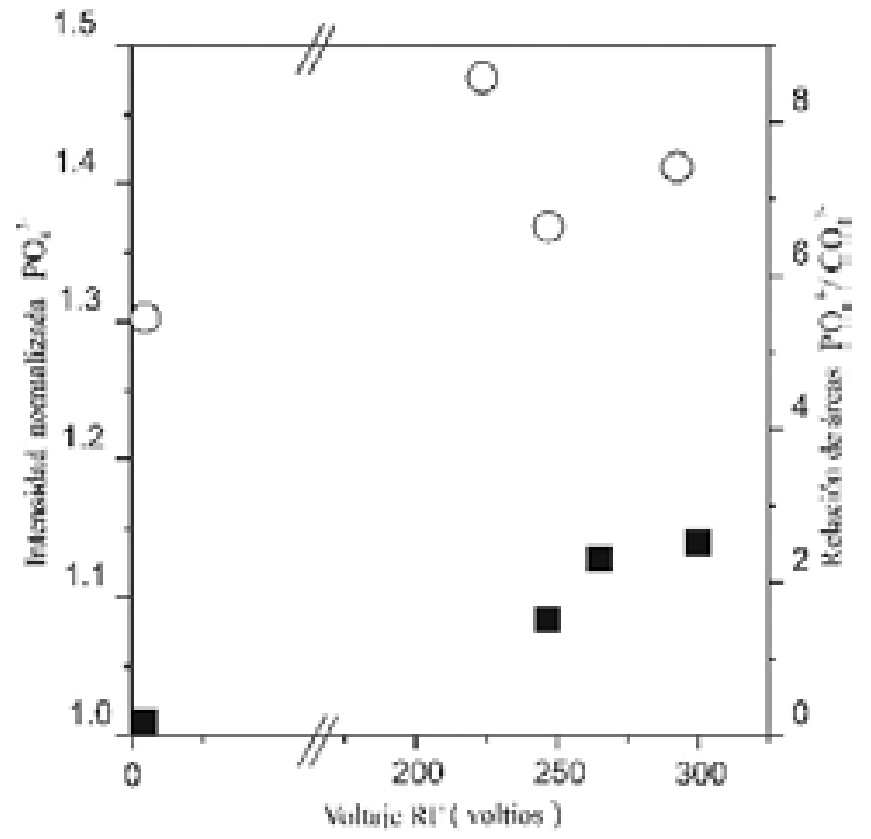

Fig. 3- Intensidad normalizada de la banda $\mathrm{PO}_{4}^{3-} v_{3}$ (cuadrados) y relación de áreas para los grupos funcionales $\mathrm{PO}_{4}^{3-} v_{3} \mathrm{y} \mathrm{CO}_{3}{ }^{2-} v_{3}$ (círculos), para diferentes valores de voltaje de radiofrecuencia.

TABLA I. VALORES DE LA RAZÓN DE Ca/P CALCULADOS MEDIANTE EDS PARA LAS CAPAS DEPOSITADAS EN ATMÓSFERA DE VAPOR DE AGUA A DIFERENTES VOLTAJES DE DESCARGA RF.

\begin{tabular}{|c|c|}
\hline Voltaje RF & $\mathrm{Ca} / \mathrm{P}$ \\
\hline 0 & $1.92 \pm 0.02$ \\
\hline 247 & $1.89 \pm 0.01$ \\
\hline 265 & $1.89 \pm 0.02$ \\
\hline 300 & $1.91 \pm 0.01$ \\
\hline
\end{tabular}

local de los recubrimientos no se ve afectada por el voltaje RF. Sin embargo, un estudio más detallado de los espectros de IR nos permite observar ciertos fenómenos ocasionados por la RF utilizada y los cuales se presentan en la Fig. 3. La intensidad de la banda de absorción de tensión asimétrica del $\mathrm{PO}_{4}^{3-}$ (I) para capas obtenidas con diferentes voltajes de RF ha sido normalizada utilizando la intensidad (Io) de dicha banda para nuestro material de referencia $\left(\mathrm{V}_{\mathrm{RF}}=0\right)$, pudiéndose observar que dicha intensidad aumenta con el voltaje aplicado, indicando un aumento en el ritmo de depósito de los recubrimientos. Por otro lado en esta misma figura está representada la relación entre las áreas de las bandas de tensión asimétrica para los grupos $\mathrm{PO}_{4}^{3-}$ y $\mathrm{CO}_{3}{ }^{2-}$ en función del voltaje de $\mathrm{RF}$, pudiéndose comprobar un ligero aumento de la concentración de grupos $\mathrm{PO}_{4}^{3-}$ a costa de los grupos $\mathrm{CO}_{3}^{2-}$, demostrando un cierto incremento en el grado de cristalinidad de los recubrimientos (19).

Un factor importante a tener en cuenta en el estudio de recubrimientos de hidroxiapatita es la relación $\mathrm{Ca} / \mathrm{P}$ de dichas capas. En este caso esta relación fue obtenida a partir de medidas de EDS, calculando previamente una recta de calibración con tres materiales de relación $\mathrm{Ca} / \mathrm{P}$ conocida. En la Tabla 1 se resumen los valores de dicha relación para las capas obtenidas por PLD. La incorporación del plasma de RF no parece afectar a esta relación ya que los valores permanecen constantes dentro del margen de error. En cuanto al análisis de estas capas por difracción de rayos X (Fig. 4), la presencia de las reflexiones (211), (112), (002) y (210) indica que la estructura apatítica está definida. En cuanto a la influencia de la RF podemos observar que a medida que ésta aumenta tiene lugar una atenuación en las reflexiones (002) y (200) e incremento de la intensidad, así como una mayor resolución e intensidad de las reflexiones (211) y (112), denotando esto último un menor contenido en grupos $\mathrm{CO}_{3}^{2-}(19,20)$, lo cual concuerda con los resultados obtenidos mediante IR (Fig. 3).

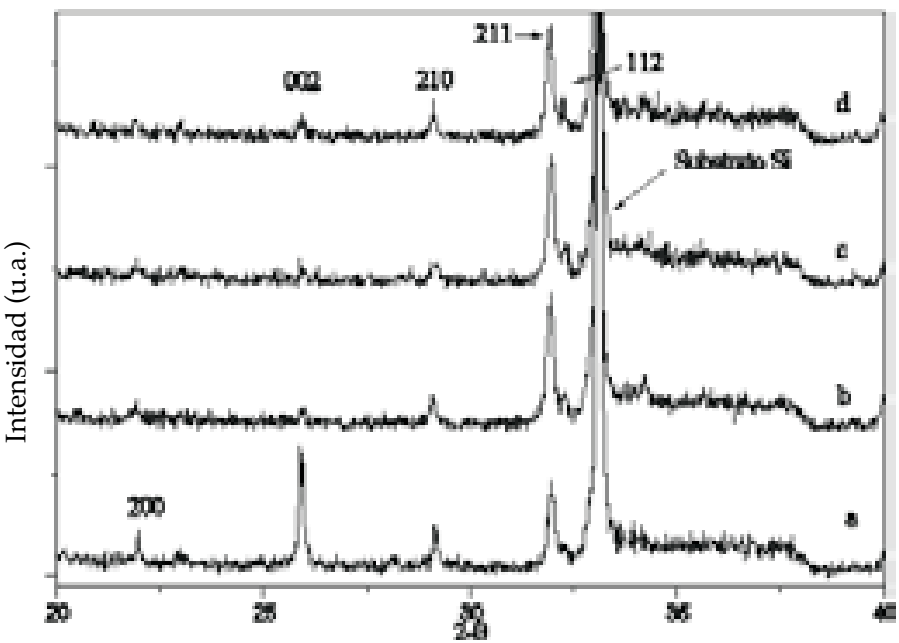

Fig. 4- Patrones de difracción de rayos $\mathrm{X}$ de las capas depositadas en atmósfera de vapor de agua a diferentes voltajes de descarga RF: a) 0 V, b) $247 \mathrm{~V}$, c) $265 \mathrm{~V}$, d) $300 \mathrm{~V}$.

\subsection{Recubrimientos con vidrio bioactivo}

La Fig. 5 muestra los espectros de absorción IR para capas de vidrio bioactivo crecidas mediante PLD sin RF en diferentes condiciones: atmósfera de $\mathrm{Ar}$, atmósfera de $\mathrm{NH}_{3}$ y vacío. Esta técnica espectroscópica es una herramienta muy útil en el 


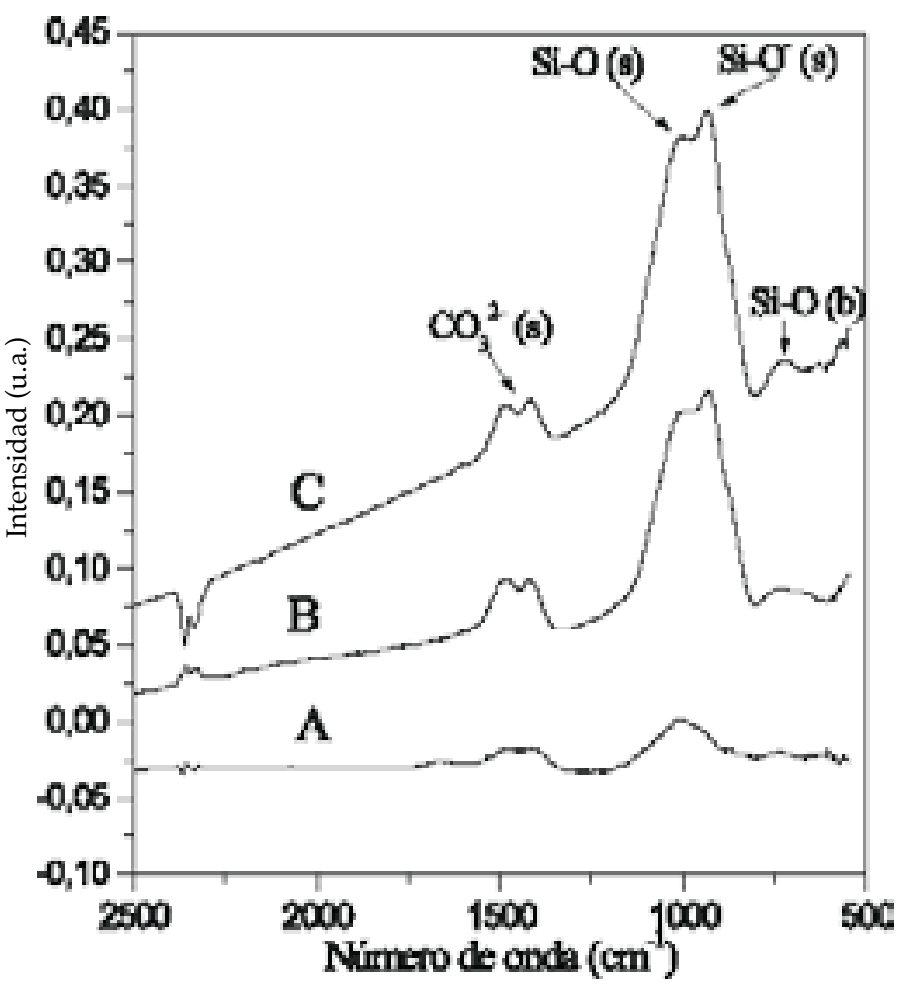

Fig. 5- Espectros FTIR de capas de vidrio bioactivo depositados en las siguientes condiciones: A) atmósfera de $\mathrm{Ar}, \mathrm{B}$ ) atmósfera de $\mathrm{NH}_{3}$ y C) vacío.

estudio de la composición de los vidrios bioactivos (21), siendo especialmente interesante la posibilidad de diferenciar entre grupos funcionales con oxígenos puente (Si-O-Si) y no puente ( $\left.\mathrm{Si}-\mathrm{O}^{-}\right)$dentro de la propia matriz de sílice, al jugar estos últimos un papel importante en la respuesta bioactiva de estos materiales (22). El espectro IR del recubrimiento depositado en vacío (material de referencia) presenta: i) una banda ancha entre 1000-1200 $\mathrm{cm}^{-1}$ correspondiente a la vibración de tensión (s) del enlace $\mathrm{Si}-\mathrm{O}-\mathrm{Si}$, ii) una banda entre $700-800 \mathrm{~cm}^{-1}$ correspondiente a la vibración de flexión (b) del enlace Si-O-Si y iii) una banda a $900-950 \mathrm{~cm}^{-1}$ correspondiente a la vibración de tensión (s) del grupo Si-O. Se observa que la utilización de atmósfera reactiva durante el proceso PLD origina modificaciones de la intensidad relativa de estas bandas. En el caso de la atmósfera de Ar se observa un ligero desplazamiento hacia números de onda mayores de la banda correspondiente a la vibración de tensión del enlace Si-O-Si, indicando cambios estructurales en la red cristalina, como ha sido descrito en el modelo de fuerzas centrales (23). La intensidad de las bandas de absorción en presencia tanto de una atmósfera de Ar como de $\mathrm{NH}_{3}$ disminuye en comparación con los depósitos realizados en vacío, lo cual indica una reducción del ritmo de depósito debida a que la presencia de una atmósfera en el proceso de ablación conlleva a la deceleración de las partículas ablacionadas (24). En el caso de la atmósfera de $\mathrm{NH}_{3}$ aparece un efecto adicional debido a que la molécula de $\mathrm{NH}_{3}$ absorbe radiación a la longitud de onda de trabajo (25), provocando que la energía que recibe el blanco sea menor.

Ha sido descrito (22) que en el caso de recubrimientos de estas características una respuesta biológica aceptable de los mismos está relacionada con la presencia de una mínima concentración de grupos $\mathrm{Si}^{-} \mathrm{O}^{-}\left(\mathrm{Si}^{-} \mathrm{O}^{-} / \mathrm{Si}-\mathrm{O}-\mathrm{Si} \geq 1\right)$. En la Tabla
TABLA II. RELACIÓN DE INTENSIDADES DE LAS BANDAS RELATIVAS A LAS VIBRACIONES DE TENSIÓN DE LOS GRUPO SI-O Y SI-O-SI.

\begin{tabular}{|c|c|}
\hline Atmósfera & $\mathrm{I}_{\text {Si-O }}-/ \mathrm{I}_{\text {Si-O-Si }}$ \\
\hline Vacío & 1,13 \\
\hline Ar & 0,73 \\
\hline Ar con RF & 0,81 \\
\hline $\mathrm{NH}_{3}$ & 1,08 \\
\hline $\mathrm{NH}_{3}$ con RF & 1,07 \\
\hline
\end{tabular}

2 se muestra el valor de esta relación de intensidades IR para las capas de vidrio bioactivo depositadas en diferentes condiciones, mostrando que: i) la relación $\mathrm{Si}-\mathrm{O} / \mathrm{Si}-\mathrm{O}-\mathrm{Si}$ es superior a 1 en depósitos realizados en vacío y atmósferas de $\mathrm{NH}_{3}$ y además en el caso de $\mathrm{NH}_{3}$ con descarga $\mathrm{RF}$, ii) los depósitos realizados en atmósfera de Ar y también en el caso de Ar con descarga de RF presentan una relación inferior a 1 , y iii) en el caso de la atmósfera de $\mathrm{NH}_{3}$ la aplicación de descarga RF no parece afectar a la relación de grupos Si-O', mientras que en el caso de la atmósfera de Ar dicha relación aumenta ligeramente. Es por esto que desde el punto de vista de la bioactividad, las condiciones más apropiadas para el crecimiento de estos recubrimientos son vacío, seguido de atmósfera de $\mathrm{NH}_{3}$, con y sin descarga de RF.

\section{CONCLUSIONES}

La atmósfera utilizada en el proceso de PLD tiene un papel muy importante en el crecimiento, enlace y estructura de los recubrimientos. Las atmósferas más apropiadas son el vapor de $\mathrm{H}_{2} \mathrm{O}$ en recubrimientos de hidroxiapatita y vacío en recubrimientos de vidrio bioactivo. La descarga de RF durante el proceso RPLD incrementa el ritmo de depósito y mejora la cristalinidad en el caso de HA, y en los recubrimientos de vidrio bioactivo no supone un incremento en la concentración de grupos Si-O'.

\section{AGRADECIMIENTOS}

Este trabajo ha sido realizado gracias al apoyo de la Xunta de Galicia (PGIDT99PX32101B, PGIDIT03TMT30101PR), Universidade de Vigo (64502I908, I418122F64102) y el Ministerio de Ciencia y Tecnología (MAT 2001-3434, MAT 2004-02791).

\section{REFERENCIAS}

1. W. Suchanek, M. Yoshimura, "Processing and Properties of Hydroxyapatitebased Biomaterials for use as Hard Tissue Replacement Implants", J. Mater. Res. Vol. 13, 1 pp. 94-117 (1998).

2. P. N. De Aza, A. H. De Aza, S. De Aza, "Crystalline Bioceramic Materials", Bol. Soc. Esp. Ceram. V. 44, 3, pp. 135-145, (2005).

3. R. Z. LeGeros, J. P. Legeros, "Dense Hydroxyapatite", pp. 139-180 en An Introduction to Bioceramics, Eds: L. L. Hench y J. Wilson, World Scientific Publishing Co., Singapore 1993.

4. L. L. Hench, O. Andersson, "Bioactive Glasses", pp. 41-62 en An Introduction to Bioceramics, Eds: L. L. Hench y J. Wilson, World Scientific Publishing Co., Singapore 1993.

5. O. H. Andersson, K. H. Karlsson, "On the bioactivity of silicate glass", Journal of Non-Crystalline Solids 129, pp. 145-151 (1991).

6. R. M. Pilliar, M. J. Filiaggi, "New calcium phosphate coating methods" pp. 165-171 en Bioceramics 6, Eds: P. Ducheyne y D. Christiansen, D. Butterworth-Heinemann, Ltd., Oxford UK 1993.

7. F. J. García-Sanz, M. B. Mayor, J. L. Arias, J. Pou, B. León, M. Pérez-Amor "A comparative study between plasma-spray and pulsed laser deposition 
techniques", J. Mater. Sci. Mater. Med. 8, pp. 861-865 (1997).

8. C. M. Cotell, D. B. Chrisey, K. S. Grabowski, J. A. Sprague, C. R. Gosset, "Pulsed Laser Deposition of Hydroxylapatite Thin Films on Ti-6Al-4V", J. App. Biomat. 3, pp. 87-93 (1992).

9. L. D. Alessio, R. Teghil, M. Zaccagnino, I. Zacardo, D. Ferro, V. Marotta, "Pulsed laser ablation and deposition of bioactive glass as coating material for biomedical applications", App. Surf. Sci. 138-139, pp. 527-532 (1999).

10. J. L. Arias, M. B. Mayor, J. Pou, B. León, M. Pérez-Amor, "Stoichometric transfer in pulsed laser deposition of hydroxylapatite", App. Surf. Sci. 154155, pp. 434-438 (2000).

11. G. Sardin, M. Varela, J. L. Morenza, "Deposition of Hysdroxyapatite coatings by laser ablation" en:, Hydroxyapatite and Related Materials, Eds: P. W. Brown, B. Constanz, CRC Press, 1994, pp. 225-230.

12. B. Mayor, J. Arias, S. Chiussi, F. García, J. Pou, B. León, M. Pérez-Amor, "Calcium phosphate coatings grown at different substrate temperatures by pulsed ArF-laser deposition", Thin Solid Films 317, pp. 363-366 (1998). 13.

13. C. M. Cotell, "Pulsed laser deposition and processing of biocompatible hydroxylapatite thin films", App. Surf. Sci, 69, pp. 140-148 (1993)

14. M. Jelínek, V. Olsan, L. Jastrabík, V. Studnicka, V. Hnatowicz, J. Kvítek, V. Havránek, T. Dostálová, I. Zergioti, A. Petrakis, E. Hontzopoulos, C. Fotakis, "Effect of processing parameters on the properties of hydroxylapatite films grown by pulsed laser deposition", Thin Solid Films 257, pp. 125-129 (1995).

15. S. Liste, P. González, J. Serra, J. P. Borrajo, S. Chiussi, B. León, M. PérezAmor, J. García López, F. J. Ferrer, Y. Morilla, M. A. Respaldiza, "The role of the reactive atmosphere in pulsed laser deposition of bioactive glass films", Thin Solid Films 453-454, pp. 219-223 (2004).

16. J. Serra, S. Liste, P. González, C. Serra, J. P. Borrajo, S. Chiussi, B. León M. Pérez-Amor, "The role of the remperature and the laser fluence on the properties of PLD bioactive glass films", Appl. Phys. A, 79, pp. 983-986, (2004).
17. J. L. Arias, F. J. García Sanz, M. B. Mayor, S. Chiussi, J. Pou, B. León, M. Pérez-Amor, "Physicochemical properties of calcium phosphate coatings produced by pulsed laser deposition at different water vapour pressures", Biomaterials 19, pp. 883-888 (1998).

18. M. B. Mayor, J. L. Arias, F. J. García-Sanz, S. Chiussi, J. Pou, B. León M. Pérez-Amor, "Hydroxyl incorporation in apatite coatings produced by pulsed laser deposition" pp. 209-213 en: Bioceramics Volume11, R. Z. LeGeros, J. P. LeGeros (Eds), World Scientific Publishing Co. Pte. Ltd. (1998).

19. R. Z. LeGeros, O. R. Trautz, J. P. LeGeros, E. Klein, “Carbonate substitution in the apatite structure (1)", Bull. Soc. Chem. Fr. Special Issue, pp. 1712-1718 (1968).

20. R. Z. LeGeros, O. R. Trautz, J. P. LeGeros, E. Klein, W. P. Shirra, “Apatite Crystallites: Effects of Carbonate on Morphology", Science 155, pp. 14091411 (1967).

21. J. Serra, P. González, S. Liste, C. Serra, S. Chiussi, B. León, M. Pérez-Amor, H.O. Ylänen, M. Hupa, "FTIR and XPS studies of bioactive silica based glasses", Journal of Non-Crystalline Solids 332, pp. $20-27$ (2003).

22. J. Serra, P. González, S.Liste, S. Chiussi, B. León, M. Pérez-Amor, H. O. Ylänen, M. Hupa, "Influence of the non-bridging oxygen groups on the bioactivity of silicate glasses", J. Mat. Sci. Mat. Med 13, pp. 1221-1225 (2002).

23. E. G. Parada, P. González, J. Pou, J. Serra, D. Fernández, B. León, M. PérezAmor, "Aging of photochemical vapor deposited silicon oxide thin films", J. Vac. Sci. Tech. A, 14 (2), pp. 436-440 (1996).

24. A. D. Sappey, N. S. Nogar, pp. 157-183 en: Laser Ablation, J. C. Millar (Eds), Springer, Germany 1994.

25. A. Lehmann, L. Shumman, K. Hübner, "Optical phonons in amorphous silicon oxides. I. Calculation of the density of states and interpretation of lo-to splittings of amorphous $\mathrm{SiO} /$ / 2.", Phys. Status Solidi 117, pp. 689-698 (1983).

Recibido: 10.01 .05

Aceptado: 31.10 .05 